\title{
A Meta-Analysis on Antibiotic Residues in Meat of Broiler Chickens in Developing Countries
}

\author{
Amine Berghiche ${ }^{1,2^{*}}$, Tarek Khenenou ${ }^{1}$ and Ibtissem Labiad ${ }^{1}$ \\ ${ }^{1}$ Institute of Agronomic and Veterinarian Sciences, University of Mohamed Cherrif Messaâdia, Souk Ahras, Algeria \\ ${ }^{2}$ Laboratory of Science and Technic of Living, Institute of Agronomic and Veterinarian Sciences, University of Mohamed Cherrif Messaâdia, \\ Souk Ahras, Algeria \\ *Corresponding author's Email: amine_berghiche@yahoo.com ; a.berghiche@ univ-soukahras.dz; ORCID: 0000-0001-6355-3591
}

Received: 02 May 2019

Accepted: 01 June 2019

\begin{abstract}
Present study consisted of performing a meta-analysis on data about the detection of antibiotic residues in chicken meat achieved from all over the researches with a wide collection and very strict selection criteria of data. The databases were searched quantitative inputs from the available scientific publications using important keywords, in order to evaluate all studies about antibiotic residue and detection methods and the reliability of the results obtained by the international researchers. Then an advanced statistical analysis on collected data was done, the first phase was a descriptive study of positive and negative cases followed by a modeling of two cases with a prediction of the values obtained and ended with an analysis of the main compounds (population size, residue detection methods and positive case rates). All performed steps are reported in detail. The results indicated that the accuracy of the detection technique is a factor that influenced on reports of residues, and caused differences in reports, there are still antibiotic residues in meat of intensively broiler chicken farms ( $45.26 \%$ of the samples analysed are positive), It is concluded that residue detection requires a high-precision qualitative analysis protocol.
\end{abstract}

Key words: Antibiotic residues, Broiler chicken, Databases, Meta-analysis.

\section{INTRODUCTION}

Antibiotic residues in food of animal origin remain a topical issue throughout the world, because the massive use of these molecules, for preventive and curative therapeutic purposes and also as food additives or growth promoters. (Preziosi et al., 2012; Berghiche et al., 2018). The lack of international regulations to control the use of antibiotics in poultry farming, especially in developing countries (possession, distribution and use are delayed), where many farmers treat their animals without knowing the conditions and quantities to administer or the withdrawal times (Mills et al., 2015).

Recent literature suggested that the term metaanalysis refers precisely to Glass (1976), who refers to the fact that results are evaluated at a higher level and from a more general perspective than the original studies. Glass thus focuses on the analysis of analyses. This method of analysis appeared in the 1970s in the fields of classical experimental sciences and psychotherapy (Webster, 2019).
From the 1990s onwards, the first meta-analyses appeared in the field of economics, particularly in environmental economics (Boyle et al., 1994; Schwartz, 1994; Smith and Huang, 1995) and also in labour economics (Jarrell and Stanley, 1990; Hedges et al., 1994; Card and Krueger, 1995).

Meta-analysis is a statistical approach, which aims to combine the results of a series of available and independent studies on the subject and then make a reproducible synthesis (Bouras et al., 2019).

The principles of meta-analysis are as follows: It should include all available trials for the treatment studied, positive and negative, published and unpublished (exhaustive research) (Marshall et al., 2019). It must be based on a precise protocol, to avoid any bias in the choice of studies included. Finally, this statistical method is applied to contextualize the probability of the outcome of all studies (Cervesato et al., 2019).

In total there are 23 studies that match the criteria for inclusion in this meta-analysis. In detail there were 17 studies in eight African countries (Congo, Sudan, 
Morocco, Nigeria, Senegal, Tunisia, Egypt and Algeria), four studies of them in three Asiatic countries (Turkey, Iraq and Palestine) and one in one European country (Bulgaria).

The uncontrolled use of antibiotics to form high-risk residues for the consumer, this work is focused on these residues through studies performed for its detection by the different methods based on a statistical analysis of the meta data available on this subject, the objective of present research was to evaluate the relationship between antibiotic therapy in broiler chickens and residues of antibiotic compounds in the white meat, a statistical analysis was based of the meta-data available on the subject.

The meta-analysis, which makes it possible to estimate this correlation by synthesizing similar studies that have common characteristics.

\section{MATERIALS AND METHODS}

\section{Experimental design}

The protocol was developed prior to the metaanalysis. It is defined in three steps described below: Identification, obtaining and selection of articles that were related to present research criteria, data extraction and statistical analysis.

\section{Defining the variables}

Different factors are likely to influence the presence of antibiotic residues in each study that included:

- The organic materials used broiler chicken meat or offal (liver, gizzard).

- Type of food (feed additive).

- Antibiotic treatments performed.

- The route and dose of administration.

- Breeding method (traditional or modern).

- Breeding condition, include the type of soil used, relative humidity, the total number of animals per farm, the surrounding temperature and the level of hygiene are all environmental factors that can vary from one farm to another.

- The methods used for the study are sources of variability.

- The age and weight of the chickens studied may also be different.

- Judgement criteria, included the endpoint was the variable measuring at the end of the experiment to define the effectiveness of the test product.

In order to understand all the factors of variation in order to define a protocol that avoids sources of bias as much as possible.

\section{Searching for articles related to the issue}

After defining the main characteristics of the studies, it is necessary to search them exhaustively.

Pubmed. Pubmed being a free, easy-to-use and very complete search database, firstly to perform present search using this site.

The search performed is as follows:

Reading of bibliographies. After sorting the articles obtained through pubmed, and reading the bibliography of each article, in order to retrieve other articles.

Google scholar. Google scholar, allowed the searching of academic work. It was used last to ensure that no studies dealing had been forgotten.

Selection of usable articles. Initially, articles were selected based on their title and abstract, when available. The rest of the selection was made after obtaining the entire items; the selected items should have certain characteristics, which are sample size, type of antibiotic sought and method used for detection.

Selection on title and summary. The first selection was made simply from the title of the articles, also keywords related to antibiotic residues in broiler chicken meat. The selected articles were further developed by reading their abstracts. A decision was taken to include only articles in English or French languages. This first phase excluded non-native language articles and studies on the detection of antibiotic residues in foodstuffs apart on white meat. Unfortunately, the reviewing of titles and abstracts sometimes leaves some doubt as to whether or not a study can be included in the meta-analysis. In this case the selection was made base on the entire article.

Selection on the entire article. The quality of some articles was not always adequate and some articles just did not meet the requirements of the meta-analysis.

The authors of this study decided that evaluate the quality of the article for including in present study base on following condition:

- Animal species has been specified, is the broiler chicken.

- The antibiotics used in poultry farming that anybody looking for them (like Tetracycline, Sulfonamides, Aminosides, Macrolide, Beta lactamine, Tylosin, Colistin etc.).

- The research method used (four boxes reference method)

Characteristics of the articles. The details of the articles, those were not essential, but interesting and allowing to explain some calculations. The exclusion criteria for each item were clearly stated, in this study 
only those concerning the detection of chloramphenicol was excluded, because this molecule is currently not commercialized (Fowler, 1992).

\section{Studies included in the meta-analysis}

The contamination of animal food origin by antibiotic residues has been reported by many authors, the selected research data are listed in the following summary table (Table 1).

Table 1. Summary table of studies concerned by the meta-analyses on antibiotic residues in meat of broiler chickens (Data included)

\begin{tabular}{llcc}
\hline Study & Authors & Years & Counrty \\
\hline 01 & Okombe et al. & 2016 & Congo \\
02 & Hind et al. & 2014 & Sudan \\
03 & Titouche et al. & 2016 & Algeria \\
04 & Karmi & 2014 & Egypt \\
05 & Mansouri & 2007 & Algeria \\
06 & Omotoso & 2015 & Nigeria \\
07 & Benghalem et al. & 2016 & Algeria \\
08 & Alambedji et al. & 2004 & Senegal \\
09 & Rezgui & 2009 & Tunisia \\
10 & Abiola & 2005 & Senegal \\
11 & N'kaya & 2004 & Senegal \\
12 & Ramdane & 2015 & Algeria \\
13 & Ben Mohand & 2008 & Algeria \\
14 & Chaiba et al. & 2017 & Morocco \\
15 & Ezenduka, et al. & 2014 & Nigeria \\
16 & Onurdağ et al. & 2013 & Turkey \\
17 & Shareef et al. & 2009 & Iraq \\
18 & Elmanama and Albayoumi. & 2016 & Palestine \\
19 & Shamsa. & 2013 & Iraq \\
20 & Al Pavlov et al. & 2008 & Bulgaria \\
21 & Berghiche et al. & 2017 & Algeria \\
22 & Berghiche et al. & 2018 & Algeria \\
\hline
\end{tabular}

\section{Studies not included in the meta-analysis}

The objective of this study was to detect antibiotic (chloramphenicol) residues in chicken liver, kidney and muscle by three methods: four box methods, high performance liquid chromatography and enzyme-linked immunosorbent assay test (Tajik et al., 2010), this study was excluded because the use of the antibiotic concerned is currently banned so the determination of its minimum residual limit is irrelevant. Chloramphenicol is a broadspectrum antibiotic active against Gram-positive and Gram-negative bacteria. It is an effective therapeutic agent for the treatment of many animal infections.
However, historical epidemiological data have shown that its use in humans may be associated with hematological disorders, including aplastic anemias. During the evaluation, it was not possible to set a threshold value base on the available data. This inability to set the threshold value and the shortcomings of the documents have led to a classification as a prohibited substance for use in food-producing animals in the European community since 1994 (Mensah, 2014).

\section{Statistical analysis}

The collected data are logged and processed using the program (Microsoft Office Excel, 2007) to perform the description and evaluation, for the advanced statistical part a various statistical software was used (XLSTAT, Past 3 and Pro Origin).

The first phase was a descriptive study of positive and negative cases followed by a modelling of two cases with a prediction of the values obtained and ended with an analysis of the main compounds (population size, residue detection methods and positive case rates), a value of $(\mathrm{P}=0.05)$ which is considered significant throughout all the results obtained

\section{RESULTS}

\section{Summary and description of the studies concerned by the meta-analysis}

In a summary table an exhaustive description of the studies already carried out in the field of detection of antibiotic residues in the chicken meat in an exhaustive way (Table 2).

\section{Prevalence of antibiotic residues across the different studies}

As shown in figure 1 , the presence of antibiotic residues with a rate of $45.30 \%$ was detected in 1144 cases out of a total of 2525 . There is a significant difference $(\mathrm{P}<0.05)$ between the study areas with the highest rate of antibiotic residues reported in Africa with 649 positive cases followed by Europe and Asia with 462.460 positive cases respectively (Nisha, 2008; Berghiche, 2019). A boxplot is a graph that gives a good indication of how the values of the data are distributed, the arrangement of the data on its variability or dispersion.

The comparison between the two distributions shows that the different results that are negative are compact (Figure 2A) and for the positive ones are dispersed (Figure 2B). 
Table 2. Recuperative table of results obtained across the different studies

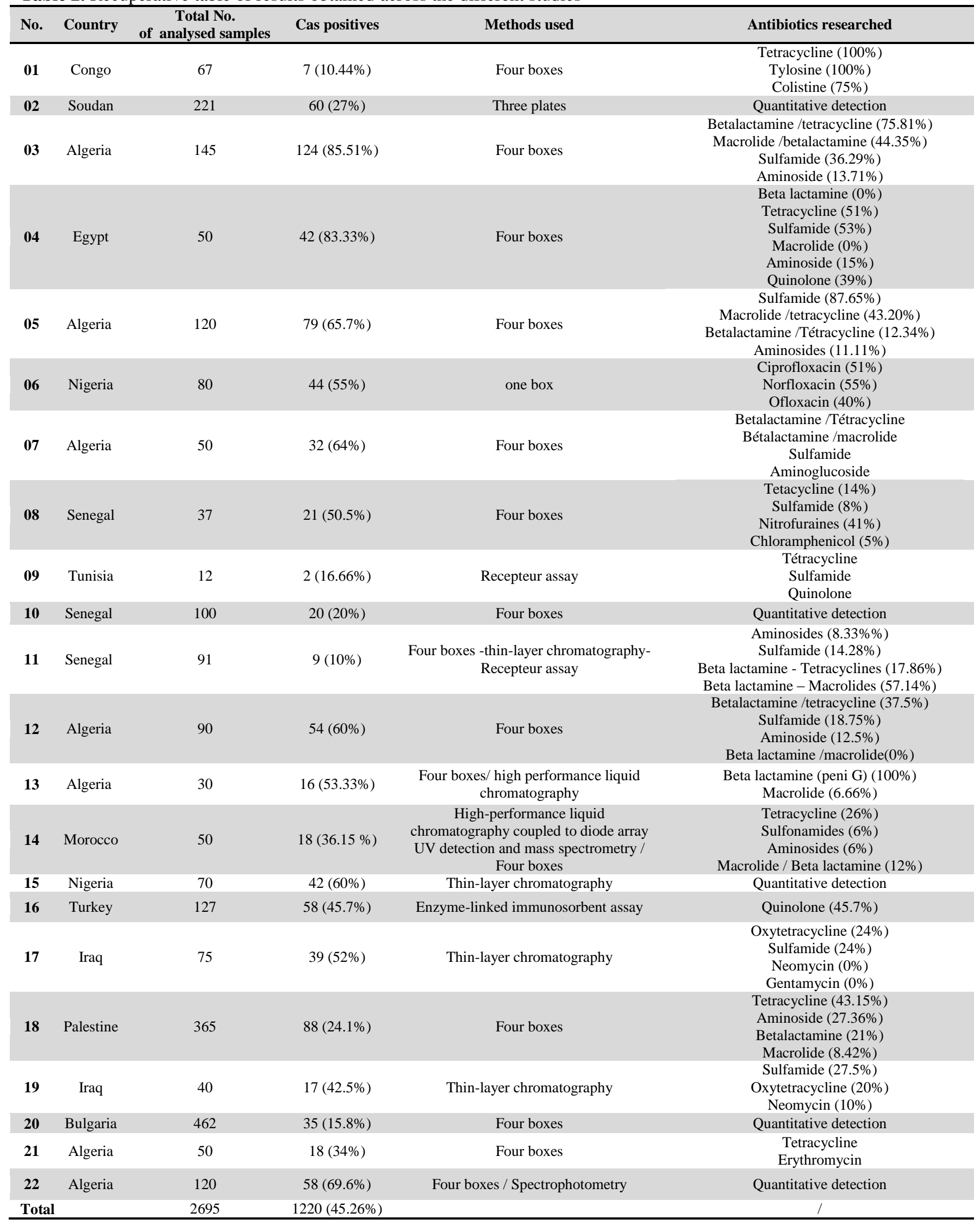




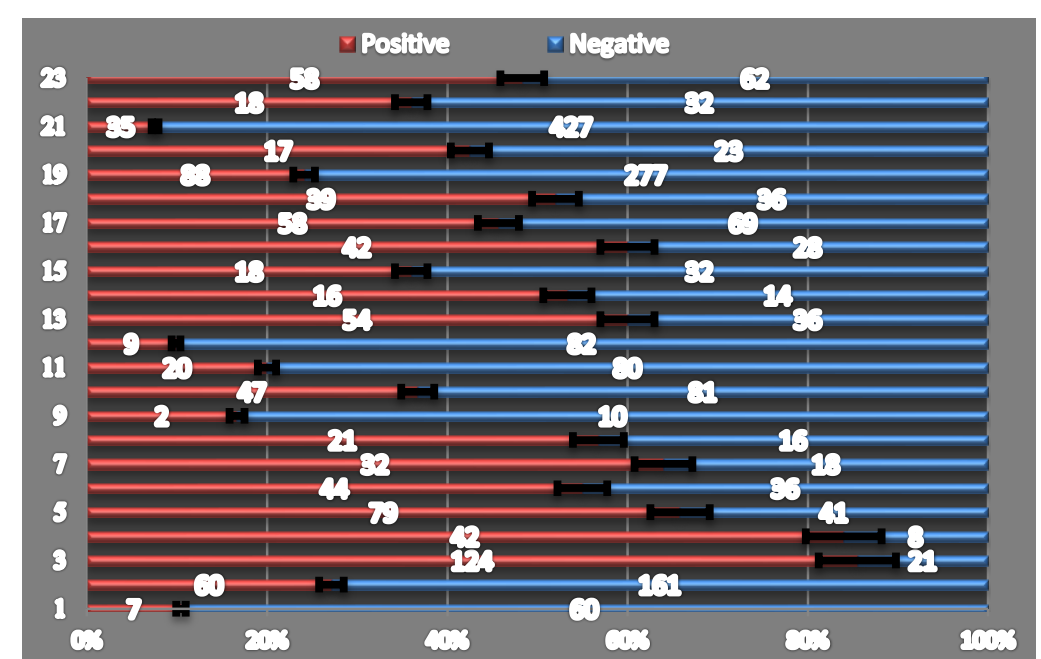

Figure 1. Recuperative results obtained of the detection of antibiotic residues used in poultry farming across the different studies in developing countries.
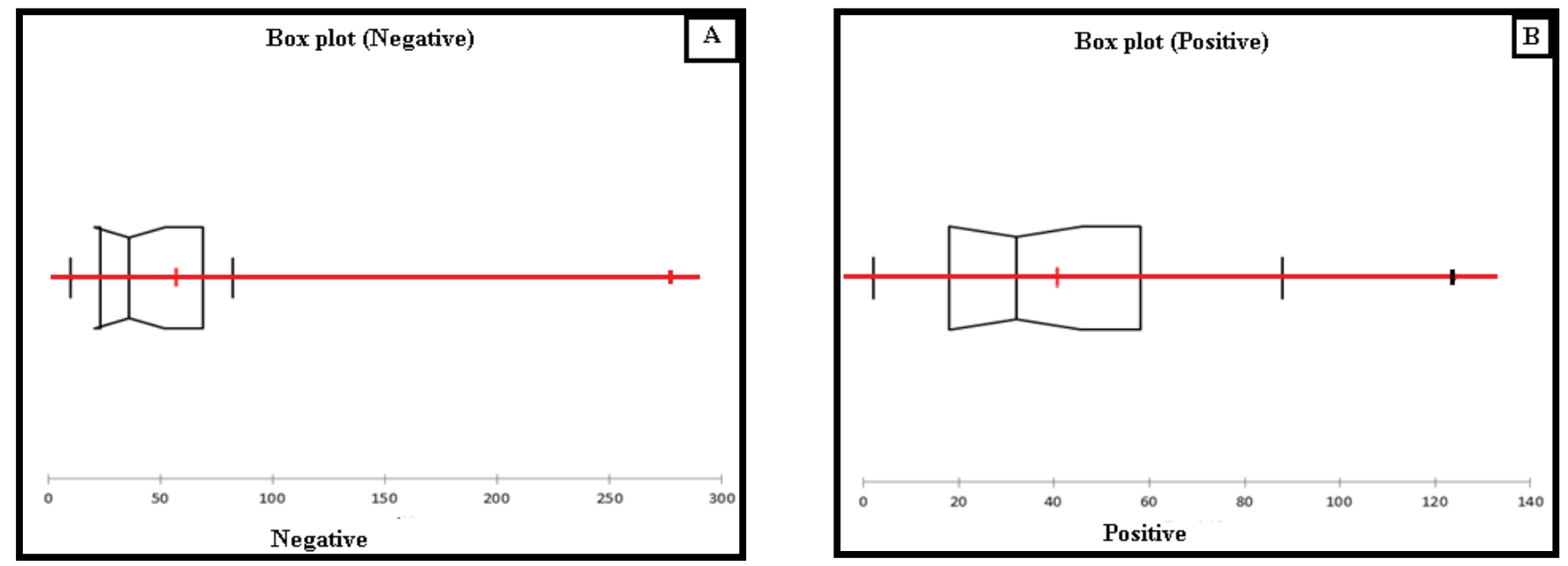

Figure 2. Results obtained from the detection of antibiotics used in poultry farming presented in Box Plot: (A) Positive cases, (B) Negative cases
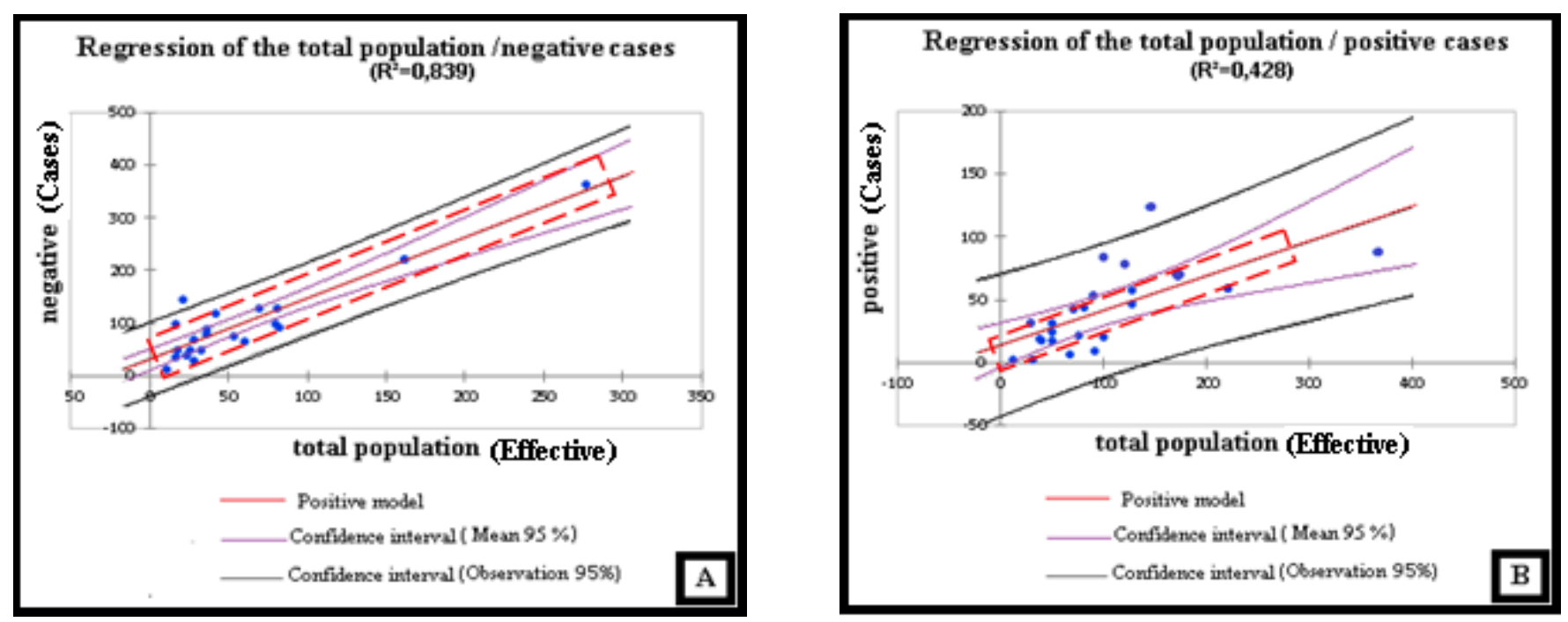

Figure 3. Modelisation of the results obtained from the detection of antibiotics used in poultry farming by linear regression in developing countries: (A) Negative cases, (B) Positive cases $\left(\mathrm{R}^{2}\right.$ : correlation coefficient) 

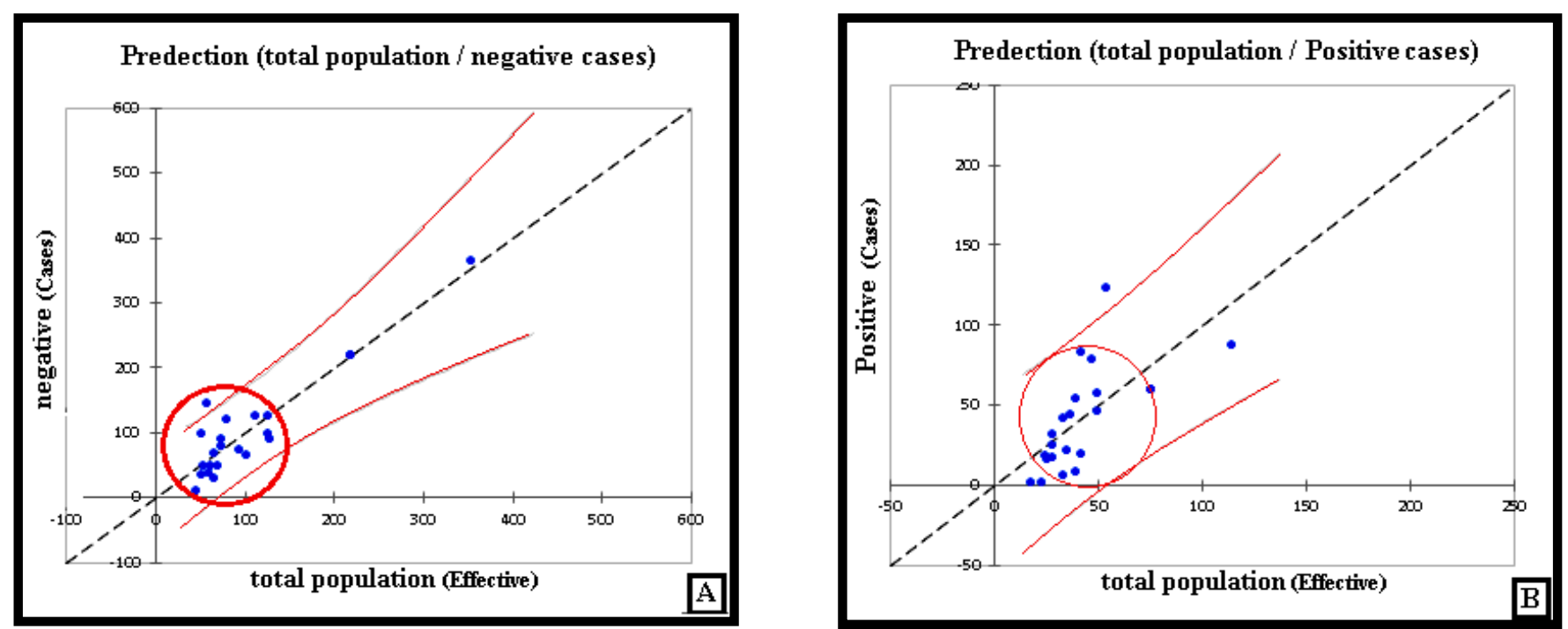

Figure 4. Results obtained from the detection of antibiotics used in poultry farming in developing countries (predetermination of the density of the values): (A) Positive cases, (B) Negative cases (Specified section: aggregation of prediction values)

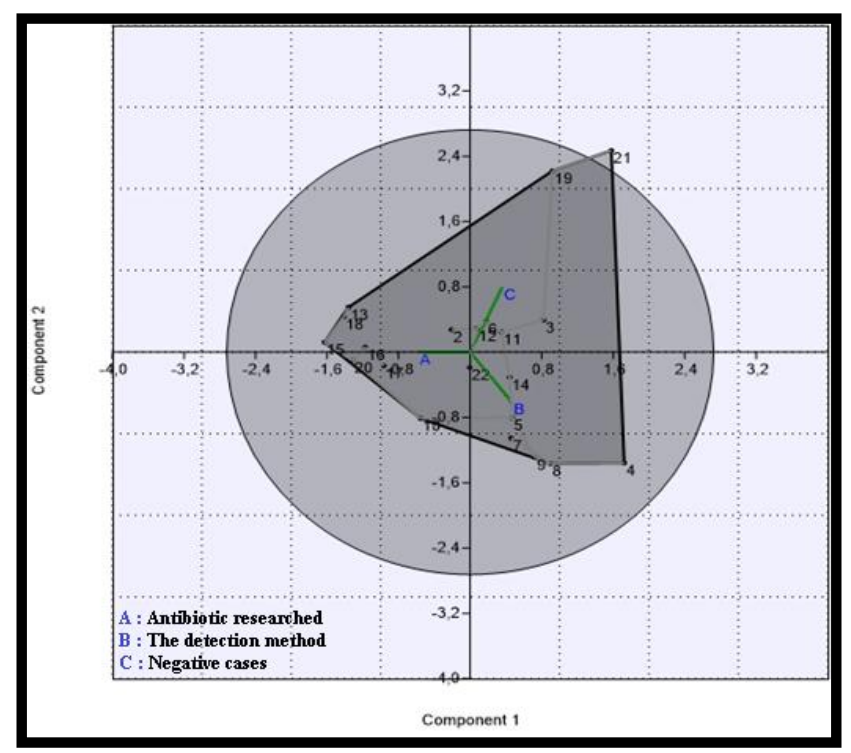

Figure 5. Factors affecting the precision of antibiotic residue detection used in poultry farming in different studies in developing countries (Analysis of the main compounds)

To properly compare the results for the two classes (positive and negative), The first step consists in developing a regression model for only 2 classes on the associated variable (Figure 3). In the second step, regression by Correlated Components is used to predict values based on detection methods to determine which ones are most reliable in the studies performed (Figure 4). Separate models were developed and then the models for the two classes were compared for the ideal explanation.

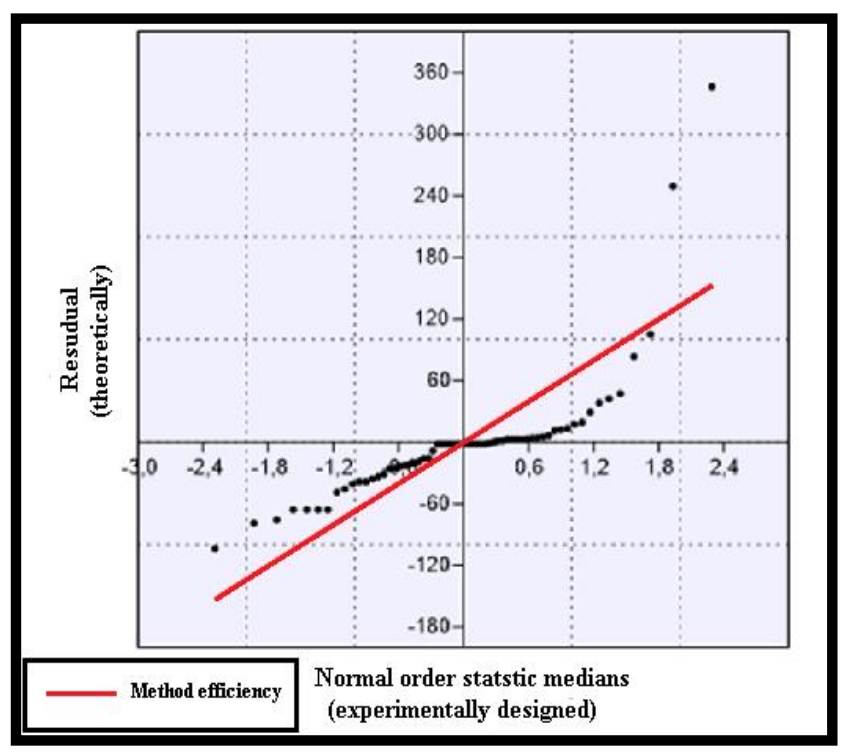

Figure 6. Effect of the Efficiency of the method in the detection of antibiotics used in poultry farming and the accuracy rate (Analysis of residual and normal order)

The use of this two-step method over two classes improves prediction compared to the traditional correlation coefficient method. The comparison between positive correlation coefficient $\left(\mathrm{R}^{2}=43 \%\right)$ and negative correlation coefficient $\left(\mathrm{R}^{2}=84 \%\right)$ since the variability of actual dependent detection (poultry samples) is explained by the variable 'detection methods' although both cases are statistically significant $\left(\mathrm{R}^{2}=0.84\right.$ and $\left.\mathrm{p}=0.05\right)$, for the confirmation of this hypothesis it is necessary to analyze 
the main compounds statistically (sample size, type of antibiotic sought and method used for detection).

The interpretation of the statistical matrices (Figures 5 and 6) shows that the detection method is the impact factor on the results, for this purpose it can be said that the methods used by the authors are not generally reliable and that the sensitivity of the techniques influenced. Through the results of these previous studies, it must be taken into consideration that the correct determination of residues in white meat involves high-precision techniques.

\section{DISCUSSION}

Among African countries, the highest percentage of residues was observed in Algeria "Souk Ahras" (100\%), while the lowest in Congo (10.7\%). (Okombe et al., 2016; Berghiche et al., 2018). In Asian countries, a high residue rate was detected in Gaza (88\%), while the lowest in Iraq $(42.5 \%)$, while in Europe, the presence of residues was reported lowest level in Bulgaria (15.8\%). (Al Pavlov et al., 2008; Shamsa, 2013; Elmanama and Albayoumi, 2016).

The high percentage of positive cases is due to noncompliance with waiting times, as well as self-medication of animals by farmers, who do not know the conditions and doses administered. This may be due to the misuse of antimicrobials probably related to animal treatment followed by insufficient withdrawal time (Corpet and Brugere, 1995; Bonfoh, 2003). Contamination of food of animal origin has been reported by many authors. Indeed, in Algeria, numerous studies have reported the presence of residues, in the Tizi Ouzou region according to Hakem et al. in 2015, sample analyses revealed the presence of 124 positive samples out of 145 collected, representing a percentage of $86.2 \%$. In the same region, Ramdane (2015) reports a $62.5 \%$ positive sample rate. In the El Taref region, Mansouri (2007) found that $65.7 \%$ of his samples containing antibiotic residues. In the Algiers region, according to Ben Mohand (2007), 3.33\% of the samples analysed contained residues of white meat.

A study conducted in northwest Algeria (Benghalem et al. (2016) found that 32 samples of chicken meat from three different regions (Tlemcen, Ain Témouchent and Sidi Bel Abbes) were positive, representing $64 \%$ of the total. At the international level, the problem of residues of veterinary drugs in foodstuffs of animal origin, particularly chicken meat, is a real problem. In Senegal, Bada-Alambedji et al. (2004) found 4 positive samples out of a total of 41 samples surveyed, representing a percentage of $9.8 \%$. Two other studies conducted in
Senegal (Dakar) by Abiola (2005) and N'kaya (2013) revealed positivity rates of $50.5 \%$ and $20 \%$ respectively. In Ankara, according to Er B1et al. (2013) $45,7 \%$ of the samples analysed were contaminated by residues. In Egypt Karmi (2014) revealed 42 positive samples out of a total of 50 samples analysed, i.e. a percentage of $83.33 \%$. In Tunisia Rezguie (2009) found 2 positive samples, i.e. a rate of $16.66 \%$. Two studies conducted in Nigeria by Omotoso (2015) and Ekene (2014) found the presence of antibiotic residues with positivity percentages of 55\% and $60 \%$ respectively. In Khartoum State (Sudan) (Hind et al., 2014) revealed that $27 \%$ of samples were positive for the presence of antibiotic residues. A study conducted in Morocco (Chaiba et al., 2017) on a total of 50 poultry meat samples revealed that 18 samples were positive for the presence of antibiotic residues, representing a rate of $36.15 \%$. Another study conducted in Palestine by Elmanama (2016) revealed 88 positive samples out of a total of 365 samples analyzed, a percentage of (24.1\%).

In Bulgaria ( $\mathrm{Al}$ Pavlov et al., 2008) found that $15.8 \%$ of samples are contaminated with antibiotic residues. Two other studies conducted in Iraq by Shareef et al. (2009) and Shamsa (2013) reported positive rates of 52\% and $42.5 \%$ respectively. In Congo (Okombe, 2016) revealed that 7 positive samples out of a total of 67 samples of chicken meat tested, i.e. a rate of $10.44 \%$. Depending on the method used, antibiotic residues were detected in 944 $(37.38 \%)$ of the cases by a microbiological inhibition method, including $660(69.91 \%)$ cases using the standard four-boxes method as the experimental protocol.

Four-boxes method has the advantage of being sensitive, with inexpensive reagents, and easy to carry out, because it allows the detection of the following four families of antibiotics, betalactamines and macrolides, betalactamines and tetracyclines; sulfamides and aminosides.

The other advantage of four boxes method is that it is less restrictive and less expensive than other microbiological methods. From our results the most important points that we have noticed, the detection of antibiotic residues in chicken meat requires firstly a high precision technique, a good understanding of the antibiotics marketed in the region that will be studied and a representative sample of white meat for a correct estimation.

\section{CONCLUSION}

The application of meta-analysis in biological domains becomes a qualitative leap, where this statistical technique 
can give reliability and meaning in life science. Despite the fact that the meta-analysis requires a lot of time and that this technique is very complicated compared to reviews. The results obtained from our meta-analysis showed that the precision of the technique for detecting antibiotic residues in chicken meat is the key factor in determining the validity of the results and the strength of its authenticity, even though the variations in the data analyzed, the application of meta-analysis allowed to summarize the results and identify defects in the detection techniques, which encourages scientists in this field to apply it to other topics.

\section{DECLARATIONS}

\section{Competing interests}

The authors have no competing interests to declare.

\section{Consent to publish}

All authors gave their informed consent prior to their inclusion in the study.

\section{Author`s contributions}

Berghiche wrote the paper, created the idea and the design the study, Labiad and Berghiche collected data, Berghiche achieves all statistical analysis and drafting of the manuscript. Khenenou and Berghiche read and approved the final manuscript.

\section{REFERENCES}

Abiola FA (2005). Antibacterial residues in the liver and gizzard of broilers in the Dakar and Thiès regions. Journal of Veterinary Medicine, 156 (5): 264-268. Available at:https://www.revmedvet.com/2005/RMV156_264_268.p df accessed on 19 May 2019.

Alambedji RB, Akakpo A, Teko-Agbo A, Chestnut B, Stevens B and Gadin B (2008). Residue control: example of antibiotics in food in Senegal [Communication]. In OIE Conference on Veterinary Drugs in Africa: Harmonization and Improvement of Registration, Distribution and Quality Control, Dakar. pp. 25-27.

Bada-Alambedji R, Cardinal E, Biagui C and Akakpo AJ (2004). Search for residues of antibacterial substance in chicken meat consumed in the Dakar region (Senegal). Bulletin of the Veterinary Academy of France, 157(2): 67-70. Available at:www.academie-veterinaire-france.fr accessed on 19 May 2019.

Ben Mohand and Chabha (2008). Master's thesis in veterinary sciences. Contribution to the study of antimicrobial residues in broiler chicken muscle.magistral thesis in vetrinary science, Option: Hygiene and food safety, National Veterinary School El-Harrach, Algeria, p. 237.

Benghalem I and Hadj Abdelkader H (2016). Contribution to the Detection of Antibiotics Residues in Liver, White Meat in North-West Algeria.Master's Degree in Quality
Management in Agro-Food Industries, Department of Agronomy, Faculty of Sciences of Nature and Life and Sciences of the Earth and the Universe,Tlemcen University, Algeria, p. 120.

Berghiche A (2019). Special Attention is Needed for Reduce Antibiotic Residue Risk in the White Meat Produced in Algeria. Journal of food quality and hazards control, 6(2): 44-44. DOI: 10.18502/jfahc.6.2.953.

Berghiche A, Khenenou T and Labiad I (2018). Antibiotics Resistance in Broiler Chicken from the Farm to the Table in Eastern Algeria. Journal of World Poultry Research, 8 (4): 95-99. PII: S2322455X1800013-8.

Berghiche A, Khenenou T and Labied I (2018). Importance of Antibiotic Residues in Food Stuffs of Avian Origin Marketed in Souk Ahras (Algerian Republic). International Journal of Veterinary Sciences and Animal Husbandry, 3(5): 5-10.

Berghiche A, Khenenou T, Bouzebda-AFri F, Lamraoui R and Labied I (2017). Detection of the antibiotic residues in broiler chickens by microbiological screening test in Algeria. Global Veterinary Journal, 19(2): 504-508. DOI: 10.5829/idosi.gv.2017.504. 508.

Bouras E, Tsilidis K K, Pounis G and Haidich AB (2019). Metaanalysis of Nutrition Studies. Analysis in Nutrition Research, 163-196. DOI: 10.1016/B978-0-12-8145562.00007-5.

Boyle KJ, Poe G L and Bergstrom JC (1994). What do we know about groundwater values? Preliminary implications from a meta-analysis of contingent-valuation studies. American Journal of Agricultural Economics, 76(5): 1055-1061 DOI: $10.2307 / 1243391$.

Card D and Krueger AB (1995). Time-series minimum-wage studies: a meta-analysis. The American Economic Review, 85(2): 238-243. Available at: https://www.jstor.org/stable/2117925 Accessed on 19 May 2019.

Cervesato I, Durgin NA, Lincoln PD, Mitchell JC and Scedrov A (1999). A meta-notation for protocol analysis. In Proceedings of the 12th IEEE Computer Security Foundations Workshop 13th The Institute of Electrical and Electronics $\quad$ Engineers, 55-69. DOI: $10.1109 / C S F W .1999 .779762$.

Chaiba AF, Rhazi Filali and A Chebaibi (2017). Investigation of Antibiotic Residues in Poultry Products in Meknes Morocco. Journal of Advances in Microbiology, 2(1): 1-8. DOI: $10.9734 / J A M B / 2017 / 31567$

Elmanama AA and Albayoumi MA (2016). High Prevalence of Antibiotic Residues among Broiler Chickens in Gaza Strip. High Prevalence of Antibiotic Residues among Broiler Chickens in Gaza Strip, 6 (4): 93-98. Available at: http://hdl.handle.net/20.500.12358/25303. Accessed on 18 May 2019.

Er B, Onurdağ FK., Demirhan B, Özgacar SÖ, Öktem AB and Abbasoğlu U (2013). Screening of quinolone antibiotic residues in chicken meat and beef sold in the markets of Ankara, Turkey. Poultry science, 92(8): 2212-2215. https://doi.org/10.3382/ps.2013-03072.

Ezenduka EV, Ike OS and Anaelom NJ (2014). Rapid detection of antimicrobial residues in poultry: A consequence of 
non-prudent use of antimicrobials. Health, 6(2): 149. DOI:http://dx.doi.org/10.4236/health.2014.62023.

Fowler NG (1992). Antimicrobials and competitive exclusion. International journal of food microbiology, 15(3-4): 277-279. DOI:https://doi.org/10.1016/0168-1605(92)90058-B.

Glass GV (1976). Primary, secondary, and meta-analysis of research. Educational researcher, 5(10): 3-8. DOI:https://doi.org/10.3102/0013189X005010003.

Hedges LV, Laine RD and Greenwald R (1994). An exchange: Part I: Does money matter? A meta-analysis of studies of the effects of differential school inputs on student outcomes. Educational researcher, 23(3): 5-14. DOI:https://doi.org/10.3102/0013189X023003005.

Hind AE, Adil M and Samah A (2014). Screening of antibiotic residues in poultry liver, kidney and muscle in Khartoum State, Sudan. Journal of Applied and Industrial Sciences, 2 (3): 116-122.

Jarrell SB and Stanley TD (1990). A meta-analysis of the unionnonunion wage gap. School of Industrial and Labor Relations $\quad$ Review, 44(1): 54-67. DOI:https://doi.org/10.1177/001979399004400104.

Karmi M (2014). Detection and presumptive identification of antibiotic residues in poultry meat by using FPT. Global journal of pharmacology, 8(2): 160-165. DOI: 10.5829/idosi.gjp.2014.8.2.82307

Marshall IJ, Marshall R, Wallace BC, Brassey J and Thomas J (2019). Rapid reviews may produce different results to systematic reviews: a meta-epidemiological study. Journal of clinical epidemiology, 109: 30-41, DOI:https://doi.org/10.1016/j.jclinepi.2018.12.015.

Nisha A R (2008). Antibiotic residues a global health hazard. Veterinary world, 1(12): 375.

Nkaya T (2004). Comparative study of the presence of antibiotic residues in the muscles of the thigh and the breast of the broiler chicken in the Dakar region. Thesis Veterinary Medicine, Dakar. Accessed on 18 May 2019.

Okombe EV, Luboya WLR, Nzuzi MG and Pongombo SC (2016). Detection of antibiotic residues in bovine and avian foodstuffs marketed in Lubumbashi (DR Congo). Journal of Applied Biosciences, 102 (1): 9763-9770. DOI:http://dx.doi.org/10.4314/jab.v102i1.11.

Omotoso AB and Omojola AB (2015). Fluoroquinolone residues in raw meat from open markets in Ibadan, Southwest, Nigeria. International Journal of Health. Animal Science and Food Safety, 2(1): 32-40. DOI:https://doi.org/10.13130/2283-3927/4739.
Pavlov A, Lashev L, Vachin I and Rusev V (2008). Residues of antimicrobial drugs in chicken meat and offals. Trakia Journal of sciences, 6(1): 23-25.

Ramdane Mohamed Said (2015). Qualitative and quantitative study of antibiotic residues in poultry meat and eggs in the Mitidja region. Use of probiotics as an alternative. Doctoral thesis, Science, Biology specialty, Faculty of biological sciences and agronomic sciences, University Mouloud Mammeri of Tizi Ouzou, Algeria, p. 254.

Rezgui A (2009). Analysis of antibiotic residues in foodstuffs in Tunisia: The tetracyclines, quinolones, and Sulphonamides. Master thesis, Biotechnology, Quality control specialty, University of Manouba, Tunisia, pp. 53-55.

Schwartz J (1994). Air pollution and daily mortality: a review and meta-analysis. Environmental research, 64(1): 36-52.

Shamsa MT (2013). Detection of the remaining effects of antibiotics in chicken meat products in Najaf province. Technical Institute in Najaf. Qadisiyah Journal of Veterinary Medicine Sciences, 12 (2): 11-15. https://doi.org/10.1006/enrs.1994.1005

Shareef AM, Jamel ZT and Yonis KM (2009). Detection of antibiotic residues in stored poultry products. Iraqi Journal of Veterinary Sciences, 23(1): 45-48.

Smith VK and Huang JC (1995). Can markets value air quality? A meta-analysis of hedonic property value models. Journal of political economy, 103(1): 209-227.

Tajick MA and Shohreh B (2006). Detection of antibiotics residue in chicken meat using TLC. International journal of poultry science, 5(7): $611-612$.

Tajik H, Malekinejad H, Razavi-Rouhani SM, Pajouhi MR, Mahmoudi R and Haghnazari A (2010). Chloramphenicol residues in chicken liver, kidney and muscle: a comparison among the antibacterial residues monitoring methods of Four Plate Test, ELISA and HPLC. Food Chem Toxicol, 48:2464-2468.

Hakem A, Titouche Y, Houali K, Yabrir B, Malki O, Chenouf N, Yahiaoui S, Labied M, Ghenim H, Kechih S et al (2013). Screening of Antibiotics Residues in Poultry Meat by Microbiological Methods. Bulletin of the University of Agricultural Sciences and Veterinary Medicine ClujNapoca. VeterinaryMedicine, 70(1): 77-82. available at:http://journals.usamvcluj.ro/index.php/veterinary/article/ view/9832/8142 accessed on 29 december 2018

Webster, GD (2019). 21 Meta-Analyses: An Introduction. Advanced Research Methods for the Social and Behavioral Sciences, p. 346. 